\title{
Wavelength-tunable waveguides based on polycrystalline organic-inorganic perovskite microwires
}

Ziyu Wang, ${ }^{\text {ał }}$ Jingying Liu, ${ }^{\text {ał }}$ Zai-Quan Xu, ${ }^{\text {a }}$ Y unzhou Xue, ${ }^{\text {a,b }}$ Liangcong Jiang, ${ }^{\text {a }}$ Jingchao Song, ${ }^{\mathrm{a}}$ Fuzhi Huang, ${ }^{\mathrm{a}}$ Yusheng Wang, ${ }^{\mathrm{b}}$ Yu Lin Zhong, ${ }^{\mathrm{a}}$ Yupeng Zhang, ${ }^{\mathrm{a}}{ }^{\mathrm{Y}}$ Yi-Bing Cheng, ${ }^{\mathrm{a}}$ and Qiaoliang Bao*b,a

${ }^{a}$ Department of Materials Science and Engineering, Faculty of Engineering, Monash University, Clayton 3800 Victoria, Australia.

${ }^{b}$ Institute of Functional Nano and Soft Material (FUNSOM), Jiangsu Key Laboratory for Carbon-Cased Functional Materials and Devices, and Collaborative Innovation Center of Suzhou Nano Science and Technology, Soochow University, Suzhou 215123, P. R China

*Corresponding authors

E-mail addresses: qlbao@suda.edu.cn, yibing.cheng@moansh.edu, yupeng.zhang@moansh.edu

${ }^{\ddagger}$ These authors contributed equally to this work. 


\begin{abstract}
Hybrid organic-inorganic perovskites have emerged as new photovoltaic materials with impressively high power conversion efficiency due to the high optical absorption coefficient and long charge carrier diffusion length. In addition to high photoluminescence quantum efficiency and chemical tunability, hybrid organic-inorganic perovskites also show intriguing potential for diverse photonic applications. In this work, we demonstrate that polycrystalline organic-inorganic perovskite microwires can function as active optical waveguides with small propagation loss. The successful production of high quality perovskite microwires with different halogen elements enables the guiding of light with different colours. Furthermore, it is interesting to find that out-coupled light intensity from the microwire can be effectively modulated by an external electric field, which behaves as an electro-optical modulator. This finding suggests the promising applications of perovskite microwires as effective building blocks in micro/nano scale photonic circuits.
\end{abstract}




\section{Introduction}

During past decade, one-dimensional (1D) micro or nano-structures based on inorganic semiconductors ${ }^{1}$, polymer ${ }^{2}$ and small molecular compounds ${ }^{3}$ have been demonstrated as effective interconnects or building blocks for development of miniaturized photonic circuits. ${ }^{4}$

${ }^{5}$ Generally, there are two types of optical interconnects, i.e., silica-based passive waveguide and luminescent material based active waveguide. The leverage of manipulation of light in miniaturized photonic integrations are more likely based on the optical active waveguides because the passive waveguide possess stringent requirements for the coupling of external light source, whereas the active waveguide can be operated upon simple light illumination. ${ }^{6}$

In order to develop active waveguides with good performance, novel optical materials with high quantum yield of photoluminescence (PL), large stimulated emission cross-section and chemically tunable light emission are desired. ${ }^{7}$ In addition, 1D crystalline structure with smooth geometric surface is normally required to maintain high PL efficiency and minimize scattering loss along the light propagating axis. ${ }^{2,7}$ Thus, a large number of studies have shown that the single crystalline 1D wires can function as efficient waveguides capable of guiding light with low losses. However, most of single crystalline microwires or nanowires are not easy to produce, which may need high temperature processes ${ }^{8}$, complicated chemical reactions $^{9}$, or precise physical deposition ${ }^{10}$. As the waveguide performance is not only limited by the crystallinity and geometry of the wires but also associated with many different physical properties of the materials, an intriguing issue is whether polycrystalline 1D materials with very high photoluminescence quantum efficiency and long photocarrier diffusion length can function as active waveguides. Obviously, this has been largely unexplored but is very meaningful, considering the easy production of polycrystalline wires. 
Recently, the hybrid organic-inorganic perovskites in the form of $\mathrm{ABX}_{3}\left(\mathrm{~A}=\mathrm{CH}_{3} \mathrm{NH}_{3}{ }^{+}, \mathrm{B}=\mathrm{Pb}^{-}\right.$ ${ }^{2+}$ or $\mathrm{Sn}^{2+}$ and $\mathrm{X}^{2} \mathrm{Cl}^{-}, \mathrm{I}^{-}$and or $\mathrm{Br}^{-}$) have been demonstrated to be promising energy materials owing to facile solution processability and superior photovoltaic performance ${ }^{11-14}$ which are correlated to high carrier mobility ${ }^{15}$, suitable direct bandgap ${ }^{16}$ and long balanced photocarrier diffusion length ${ }^{17}$. Due to large optical gain ${ }^{18-20}$ and high quantum yield ${ }^{21,22}$, hybrid perovskites have also been demonstrated as novel semiconductor materials for photonic applications. Furthermore, it is noteworthy that, even for the polycrystalline perovskite, the PL quantum yield efficiency is still extremely high and carrier diffusion length more than 1 $\mu \mathrm{m}$ could be achieved, which can fully meet the requirements in photonic applications in terms of light guiding. In addition to excellent optical properties and strong light-matter interactions, the halogen elemental composition in perovskite could be substituted by reacting with different $\mathrm{CH}_{3} \mathrm{NH}_{3}(\mathrm{MA}) \mathrm{X}\left(\mathrm{X}=\mathrm{Cl}^{-}, \mathrm{Br}^{-}\right.$or $\left.\mathrm{I}^{-}\right)$, affording the broad wavelength tunability ${ }^{23}$,

${ }^{24}$. Considering with all above mentioned properties, the polycrystalline perovskite 1D wires are expected to be a new type of optical waveguide with different operation regime.

In this work, polycrystalline perovskite microwires with controlled halogen elements were successfully synthesised via a combined solution process and vapour phase conversion method. Owing to the high PL quantum yield, it is interesting to find that polycrystalline perovskite wires show unexpected waveguide performance with a small optical loss comparable to that of single crystalline wires. More importantly, the sizable band structure in perovskites brings them broad wavelength tunability covering the visible to near-infrared range.

\section{Experimental Section}

The hybrid perovskite nanowires were prepared by two steps. Firstly, $0.5 \mathrm{~g} \mathrm{PbI}_{2}$ powders (Sigma-Aldrich) were dissolved completely in $5 \mathrm{~mL} N, N$-Dimethylmethanamide (DMF, 
Sigma-Aldrich) solution at $90{ }^{\circ} \mathrm{C}$ for 1 hour. After 12 hours standing time, the $\mathrm{PbI}_{2}$ microwires precipitated while adding deionized water to $\mathrm{PbI}_{2} / \mathrm{DMF}$ solution. $\mathrm{PbI}_{2}$ wire suspension was spin-coated onto a silicon oxide substrate or cover glass (thickness: $0.17 \mathrm{~mm}$ ) to achieve random orientations and distribution for further conversion. In the second step, the $\mathrm{CH}_{3} \mathrm{NH}_{3} \mathrm{I}$ (MAI) powders ${ }^{25}$, were placed at the central of a chemical vapour deposition (CVD) furnace while the as-grown $\mathrm{PbI}_{2}$ microwires on silicon oxide or cover glass substrates were mounted downstream of the carrier gases. The central heating zone was lift to $120{ }^{\circ} \mathrm{C}$ and maintained at this temperature under low pressure condition (40-50 torr) for 40 minutes. Ar and $\mathrm{H}_{2}$ were introduced into the quartz tube as carrier gas with flow rate of $35 \mathrm{sccm}$ and $15 \mathrm{sccm}$, respectively, during the whole conversion process. The furnace was then cooled down to room temperature with the lid closed at a cooling rate of $5^{\circ} \mathrm{C} / \mathrm{min}$. The composition of $\mathrm{MAPbBr}_{\mathrm{x}} \mathrm{I}_{3-\mathrm{x}}$ can be tuned by controlling the conversion time (15 minutes to 2 hours) when the microwires were annealed at $120{ }^{\circ} \mathrm{C}$ under excessive $\mathrm{MABr}$ atmosphere under low pressure (40-50 torr). Longer annealing time results in higher iodine substitution.

The morphologies of the hybrid perovskite microwires were characterized using atomic force microscopy (AFM, Bruker, Dimension Icon SPM) and scanning electron microscopy (SEM, FEI Nova NanoSEM 450). The microstructure of as-grown $\mathrm{PbI}_{2}$ microwires was investigated with transmission electron microscopy (TEM, FEI Tecnai G2 T20). The PL spectra, waveguide properties and electro-optical modulation (EOM) measurements of perovskite microwares were carried out with a confocal microscope system (WITec, alpha 300R) with a $100 \times$ objective at ambient environment. A green laser $(532 \mathrm{~nm})$ was used to excite the $\mathrm{MAPbI}_{3}, \mathrm{MAPbBr}_{\mathrm{x}} \mathrm{I}_{3-\mathrm{x}}$ and $\mathrm{MAPbBr}_{3}$ microwires. The electro-optical modulator was fabricated with a lithography-free method: firstly, perovskite microwires were grown on $\mathrm{SiO}_{2}$ (thickness: $\sim 300 \mathrm{~nm}$ )/p-Si substrate. Following, a $\mathrm{Si}_{3} \mathrm{~N}_{4}$ shadow mask designed and milled with focus ion beam (FEI Helios, Dual Beam), was used to define the electrodes. Last, the 
sample and the mask were aligned with a customized X-Y-Z micro-poitioner before depositing $\mathrm{SiO}_{2} / \mathrm{Ti} / \mathrm{Au}(200 \mathrm{~nm} / 5 \mathrm{~nm} / 100 \mathrm{~nm})$ with electron beam evaporator.

\section{Results and discussion}

The hybrid organic-inorganic perovskite microwires were prepared by a two-step method, as schematically shown in Fig. 1a. The high quality $\mathrm{PbI}_{2}$ microwires were firstly synthesised using a solution process. In general the solvent medium and temperature in solution process are key factors that affect the crystallinity, morphology and texture of the precipitated crystals. ${ }^{21}$ Owing to the different solubility of $\mathrm{PbI}_{2}$ in $\mathrm{DMF}$ and water, we developed a new crystallization method to prepare $\mathrm{PbI}_{2}$ microwires. By controlling the $\mathrm{DMF} /$ water ratio and inversing the growth temperature, the resulting $\mathrm{PbI}_{2}$ microwires with different diameters and lengths could be produced. The low magnification TEM and optical images of $\mathrm{PbI}_{2}$ microwire are shown in Fig. 1b and 1c (top), revealing smooth surface and highly crystalline structure with a length up to $80 \mu \mathrm{m}$. The corresponding selected area electron diffraction (SAED) pattern shown in inset of Fig. $1 \mathrm{~b}$ confirms the single crystalline character of the $\mathrm{PbI}_{2}$ microwire which grows along the $[010]$ direction. ${ }^{26}$ The $\mathrm{MAPbI}_{3}$ perovskite microwires were then formed through intercalating the MAI molecules into the interval sites of $\mathrm{PbI}_{6}$ octahedrons layers. The $\mathrm{MAPbI}_{3}$ perovskites still maintains the wire shape as displayed in Fig. 1c (bottom), indicating the good morphology retention after vapour phase conversion. The slight difference in optical contrast between the perovskite and $\mathrm{PbI}_{2}$ microwires (Fig. 1c) could be attributed to the different light absorbance in these two materials as well as different light reflection from wire surface. High magnification SEM image indicates that the perovskite wire is polycrystalline with relative rough surface, as shown in Fig. 1d. The AFM results shown in Fig. 1e depict that the as-synthesised $\mathrm{MAPbI}_{3}$ microwire has a rough surface but a relatively uniform diameter of about $300 \mathrm{~nm}$ along the long axis. 
Even with a relatively rough surface, the polycrystalline organic-inorganic hybrid perovskite microwires exhibit an impressively good optical waveguide performance which is comparable to single crystal semiconductor microwires. In order to investigate the waveguide properties, PL spectroscopy as well as imaging measurements were implemented by locally exciting the wire with a fine focused laser, as schematically illustrated in Fig. 2a. Fig. 2b shows a set of dark field images of a $\mathrm{MAPbI}_{3}$ microwire with laser spot $(532 \mathrm{~nm})$ moving along the microwire. Besides the emission from excitation position, red colour PL was observed at the end of the microwire as well, suggesting typical waveguiding behaviour. It is interesting to find that there is not too much redundant PL emission along the polycrystalline microwire which has a rough surface with many grain boundaries. Alternatively, there is strong out-coupled PL at rightmost tip, suggesting a directional propagation of downconverted light. Due to the long carrier lifetime, it seems that the photocarriers prefer to propagate through the grain boundaries rather than recombine at those boundaries, and eventually recombine at the wire tip. The out-coupled light is weakened with the increase in propagation distance. Fig. 2c shows the change of out-coupled PL spectra as a function of distance between local excitation position and one end of microwire. These PL spectra demonstrate a clear PL intensity decay while prolonging the propagation distance, which is due to the propagation loss. In addition, the out-coupled PL spectra are gradually red-shifted towards the locally excited emission as the propagation distance increases (Fig. 2d), which reveals the typical character of re-absorption and re-emission in active waveguide when the guided light propagates. ${ }^{7,21,27}$

One of the most interesting nature of organic-inorganic hybrid perovskite is that the halogen elemental composition could be substituted by reacting with different $\mathrm{MAX}\left(\mathrm{X}=\mathrm{Cl}^{-}, \mathrm{Br}^{-}\right.$or $\left.\mathrm{I}^{-}\right)$, which could induce a changeable band structure and consequently tunable optical properties. The SEM morphologies of $\mathrm{MAPbBr}_{3}, \mathrm{MAPbBr}_{\mathrm{x}} \mathrm{I}_{3-\mathrm{x}}$ and composition-graded perovskite 
microwires are presented in Fig. 3a-c, respectively. The 1D morphology of perovskite microwire is maintained after vapour phase anion-substitution reaction while the diameter of microwire slightly changes due to the radius difference of halogen atoms. Fig. 3d-f depict three sets of dark field images of $\mathrm{MAPbBr}_{3}, \mathrm{MAPbBr}_{\mathrm{x}} \mathrm{I}_{3-\mathrm{x}}$ and composition-graded microwires. As expected before, the strong PL emission was observed both at the local excitation point and the wire tip for all three types of samples. It is interesting to observe green and orange colour emissions from the tips of $\mathrm{MAPbBr}_{3}$ and $\mathrm{MAPbBr}_{\mathrm{x}} \mathrm{I}_{3-\mathrm{x}}$ wires, respectively. For the composition-graded perovskite microwire, it is notable that the locally excited emission changes from green to red while changing the excitation position, whereas only red colour light is out-coupled at rightmost tip. As schematically shown in Fig. 3g, the emitted light actively propagates along the direction with decreasing bandgap within the composition-graded nanowires. The important feature of this type of waveguide is that the propagation loss caused from self-absorption is significantly reduced, making it suitable for wavelength-selective devices. The locally excited emission further confirms the successful synthesis of the composition-graded perovskite through the well-controlled annealing process. Through anion exchange, the band structure and PL emission of perovskite microwires are thus tunable, leading to the wavelength-tunable waveguide properties.

Fig. 3h depicts the PL spectra of the as-synthesized $\mathrm{MAPbI}_{3}$ (red curve), $\mathrm{MAPbBr}_{\mathrm{x}} \mathrm{I}_{3-\mathrm{x}}$ (orange curve), $\mathrm{MAPbBr}_{3}$ (green curve) microwires at room temperature. The peaks of PL emission of $\mathrm{MAPbBr}_{3}, \mathrm{MAPbBr}_{\mathrm{x}} \mathrm{I}_{3-\mathrm{x}}$ and $\mathrm{MAPbI}_{3}$ microwires appear at approximately 535 $\mathrm{nm}, 605 \mathrm{~nm}$ and $770 \mathrm{~nm}$, respectively, which are consistent with previous studies. ${ }^{27} \mathrm{In}$ comparison to $\mathrm{MAPbI}_{3}$, the PL spectrum is broadened and blue-shifted in $\mathrm{MAPbBr}_{\mathrm{x}} \mathrm{I}_{3-\mathrm{x}}$, possibly due to the nature of alloying composition. It is noteworthy that the $\mathrm{MAPbI}_{3}$ microwire shows the best waveguide properties among these hybrid perovskite microwires, which may be attributed to less defects and composition inhomogeneity introduced during 
anion-exchange process. This is experimentally confirmed by further quantitative interpretation which plots relative PL intensity ratio with respect to the propagation length, as shown in Fig. 3i. The ratio of out-coupled PL intensity $\left(I_{\text {out }}\right)$ to the locally excited PL intensity $\left(I_{\text {in }}\right)$ can be well fitted to an exponential decay function expressed by $I_{\text {out }} / I_{\text {in }}=\exp (-$ $\alpha x$ ), where $\alpha$ is a fitting parameter and $x$ is the propagation length. The propagation loss for a given propagation length is thus described as $L=-10 \log [\exp (-\alpha x)] / x .^{28,29}$ It hence can be estimated that the resulting fitting parameter $(\alpha)$ for $\mathrm{MAPbI}_{3}$ is approximately $690 \mathrm{~cm}^{-1}$ and

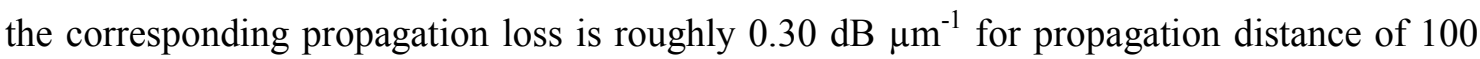
$\mu \mathrm{m}$. The propagation loss is at the same magnitude of order as those of typical single crystalline organic semiconductor microwires ${ }^{30}$, even though $\mathrm{MAPbI}_{3}$ microwire has polycrystalline structure. This is due to the long diffusion length in perovskite, in which, both the electrons and holes diffusion length exceed $1 \mu \mathrm{m}$, resulting in a large stokes shift and low re-absorption of propagating light. In conclusion, the perovskite microwires prepared by the controlled anion-exchange annealing routes can be unitized to develop wavelength tunable waveguides operating in a broad wavelength range from visible to near-infrared $(535 \sim 770 \mathrm{~nm})$.

In order to explore the change of guiding light through microwires under external electrical field, an EOM was fabricated with a parallel-plate capacitor structure. The schematic and optical images of this device are shown in Fig. $4 \mathrm{a}$ and $4 \mathrm{~b}$. The perovskite microwire is sandwiched by two silicon oxide layers so as to be electrically insulated from top (gold) and back (silicon) electrodes. As shown in Fig. 4c, it is found that increased bias voltage (from 0 $\mathrm{V}$ to $8 \mathrm{~V}$ ) leads to a linearly substantial decline in the intensity of far-field PL from the outcoupled end of the microwire. The significant decrease of the out-coupled PL intensity at the bias of $8 \mathrm{~V}$ gives a large modulation of $70 \%$ of the out-coupled PL intensity. As shown in Fig. $4 \mathrm{~d}$, the simulated local electrical field distribution within the device can reach $\sim 8 \times 10^{4} \mathrm{~V} \mathrm{~cm}^{-1}$ 
in order to achieve a modulation of $70 \%$. The large intensity modulation in the perovskite based EOM should be attributed to the ions migration caused by poling effect under the electric field. In general, electro-migration of anions (halogen ions) or cations $\left(\mathrm{MA}^{+}\right)$could induce the changes of microstructure as well as electronic structure, thus affecting the absorption coefficient or refractive index ${ }^{31,32}$. As a result, significant decrease of the outcoupled PL intensity at the end of the microwire could be observed with increasing bias voltage. The mechanism responsible for the optical property change upon poling is nontrivial and worthy further investigation.

\section{Conclusions}

In summary, optical waveguiding was observed in polycrystalline hybrid organic-inorganic perovskite microwires with different compositions. The wavelength tunability of the perovskite microwires has been demonstrated in the spectral range from visible to near infrared. Owing to the extremely high quantum efficiency and ultralong carrier migration distance, this emerging class of photovoltaic materials with a 1D-morphology show excellent waveguiding properties with a small propagation loss of $0.30 \mathrm{~dB} / \mu \mathrm{m}$. An EOM based on a hybrid organic-inorganic perovskite microwire was fabricated, in which a large intensity modulation of $70 \%$ under low electrical field strength was demonstrated. The waveguiding and modulation functions developed in this work could open up new opportunities for exploring new photonic applications based on perovskite.

\section{Acknowledgements}

This work was supported by ARC DECRA (DE120101569) and DSI top-up grant, DP (DP140101501), Engineering Seed Fund at Monash University. Z. Wang acknowledges 
support from the MATCM scholarship. Q. Bao also acknowledges the support from 863 Program (Grant No. 2013AA031903), the youth 973 program (2015CB932700), the National Natural Science Foundation of China (Grant No. 91433107, 51222208, 51290273). This work was performed in part at the Melbourne Centre for Nanofabrication (MCN) in the Victorian Node of the Australian National Fabrication Facility (ANFF). 


\section{References}

1. T. Zhai, L. Li, Y. Ma, M. Liao, X. Wang, X. Fang, J. Yao, Y. Bando and D. Golberg, Chemical Society Reviews, 2011, 40, 2986-3004.

2. D. O'Carroll, I. Lieberwirth and G. Redmond, Nature nanotechnology, 2007, 2, 180-184.

3. Y. S. Zhao, H. Fu, A. Peng, Y. Ma, D. Xiao and J. Yao, Advanced materials, 2008, 20, 2859-2876.

4. S. A. Maier, P. G. Kik, H. A. Atwater, S. Meltzer, E. Harel, B. E. Koel and A. A. Requicha, Nat Mater, 2003, 2, 229-232.

5. S. I. Bozhevolnyi, V. S. Volkov, E. Devaux, J. Y. Laluet and T. W. Ebbesen, Nature, 2006, 440, 508-511.

6. Y. S. Zhao, H. Fu, A. Peng, Y. Ma, Q. Liao and J. Yao, Accounts of chemical research, 2009, 43, 409-418.

7. Q. Bao, B. M. Goh, B. Yan, T. Yu, Z. Shen and K. P. Loh, Advanced materials, 2010, 22, 36613666.

8. A. Pan, D. Liu, R. Liu, F. Wang, X. Zhu and B. Zou, Small, 2005, 1, 980-983.

9. Y. L. Lei, Y. Jin, D. Y. Zhou, W. Gu, X. B. Shi, L. S. Liao and S. T. Lee, Advanced materials, 2012, 24, 5345-5351.

10. J. Xu, X. Zhuang, P. Guo, Q. Zhang, W. Huang, Q. Wan, W. Hu, X. Wang, X. Zhu and C. Fan, Nano letters, 2012, 12, 5003-5007.

11. C. Kagan, D. Mitzi and C. Dimitrakopoulos, Science, 1999, 286, 945-947.

12. M. A. Green, A. Ho-Baillie and H. J. Snaith, Nature Photonics, 2014, 8, 506-514.

13. Y. Wang, Y. Zhang, Y. Lu, W. Xu, H. Mu, C. Chen, H. Qiao, J. Song, S. Li and B. Sun, Advanced Optical Materials, 2015, DOI: 10.1002/adom.201500150.

14. W. S. Yang, J. H. Noh, N. J. Jeon, Y. C. Kim, S. Ryu, J. Seo and S. I. Seok, Science, 2015, 348, 1234-1237.

15. G. Hodes, Science, 2013, 342, 317-318.

16. M. M. Lee, J. Teuscher, T. Miyasaka, T. N. Murakami and H. J. Snaith, Science, 2012, 338, 643647.

17. S. D. Stranks, G. E. Eperon, G. Grancini, C. Menelaou, M. J. P. Alcocer, T. Leijtens, L. M. Herz, A. Petrozza and H. J. Snaith, Science, 2013, 342, 341-344.

18. J. H. Im, C. R. Lee, J. W. Lee, S. W. Park and N. G. Park, Nanoscale, 2011, 3, 4088-4093.

19. J.-H. Im, J. Luo, M. Franckevičius, N. Pellet, P. Gao, T. Moehl, S. M. Zakeeruddin, M. K. Nazeeruddin, M. Grätzel and N.-G. Park, Nano letters, 2015, 15, 2120-2126.

20. H. Zhu, Y. Fu, F. Meng, X. Wu, Z. Gong, Q. Ding, M. V. Gustafsson, M. T. Trinh, S. Jin and X. Y. Zhu, Nat Mater, 2015, 14, 636-642.

21. E. Edri, S. Kirmayer, M. Kulbak, G. Hodes and D. Cahen, The Journal of Physical Chemistry Letters, 2014, 5, 429-433.

22. A. Wakamiya, M. Endo, T. Sasamori, N. Tokitoh, Y. Ogomi, S. Hayase and Y. Murata, Chemistry Letters, 2014, 43, 711-713.

23. J. H. Noh, S. H. Im, J. H. Heo, T. N. Mandal and S. I. Seok, Nano letters, 2013, 13, 1764-1769.

24. J. Xing, X. F. Liu, Q. Zhang, S. T. Ha, Y. W. Yuan, C. Shen, T. C. Sum and Q. Xiong, Nano letters, 2015, 15, 4571-4577.

25. M. Xiao, F. Huang, W. Huang, Y. Dkhissi, Y. Zhu, J. Etheridge, A. Gray - Weale, U. Bach, Y. B. Cheng and L. Spiccia, Angewandte Chemie, 2014, 126, 10056-10061.

26. J. Xing, X. F. Liu, Q. Zhang, S. T. Ha, Y. W. Yuan, C. Shen, T. C. Sum and Q. Xiong, Nano Letters, 2015, 15, 4571-4577.

27. Z.-K. Tan, R. S. Moghaddam, M. L. Lai, P. Docampo, R. Higler, F. Deschler, M. Price, A. Sadhanala, L. M. Pazos and D. Credgington, Nature nanotechnology, 2014, 9, 687-692.

28. D. O'Carroll, I. Lieberwirth and G. Redmond, Small, 2007, 3, 1178-1183.

29. K. Takazawa, J.-i. Inoue and K. Mitsuishi, Nanoscale, 2014, 6, 4174-4181.

30. C. Zhang, Y. S. Zhao and J. Yao, Physical Chemistry Chemical Physics, 2011, 13, 9060-9073. 
31. Z. Xiao, Y. Yuan, Y. Shao, Q. Wang, Q. Dong, C. Bi, P. Sharma, A. Gruverman and J. Huang, Nature materials, 2015, 14, 193-198.

32. Y. Yuan, J. Chae, Y. Shao, Q. Wang, Z. Xiao, A. Centrone and J. Huang, Advanced Energy Materials, 2015, 5, 1500615. 
(a)
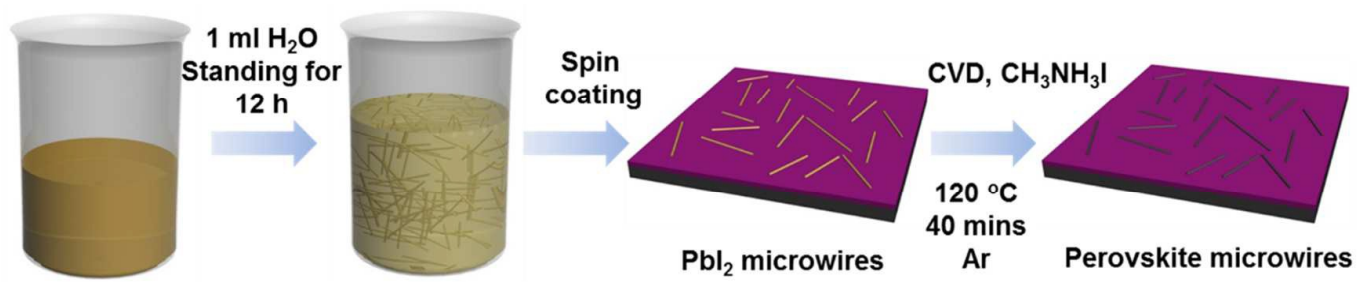

$\mathrm{Pbl}_{2}$-DMF solution (5 ml) $90^{\circ} \mathrm{C}, 1 \mathrm{~h}$

(b)

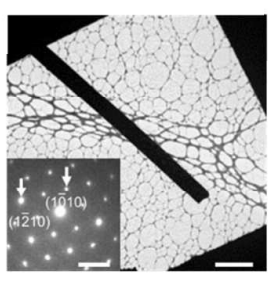

$\mathrm{Pbl}_{2}$ microwires

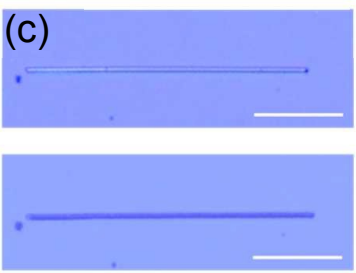

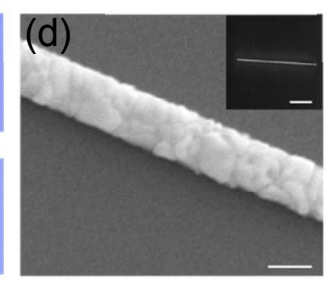

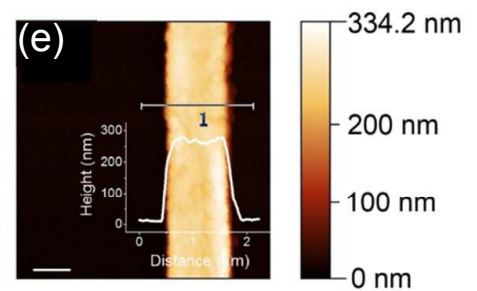

Figure 1 (a) Schematic illustration of solution process to fabricate $\mathrm{PbI}_{2}$ microwires and vapour phase conversion process to transfer $\mathrm{PbI}_{2}$ into hybrid perovskite microwires. (b) TEM image of a typical $\mathrm{PbI}_{2}$ microwire. Scale bar: $5 \mu \mathrm{m}$. Inset: corresponding SEAD pattern; scale bar: $5 \mathrm{~nm}^{-1}$. (c) Optical images of $\mathrm{PbI}_{2}$ (top) and perovskite (bottom) microwires. Scale bar: $20 \mu \mathrm{m}$. (d) High magnification SEM image of perovskite microwire. Scale bar: $500 \mathrm{~nm}$. Inset: low magnification SEM of the perovskite microwire. Scale bar: $20 \mu \mathrm{m} \quad$ (e) AFM height image of a perovskite micowire. Scale bar: $1 \mu \mathrm{m}$. Inset: height profile along line 1 . 
(a)

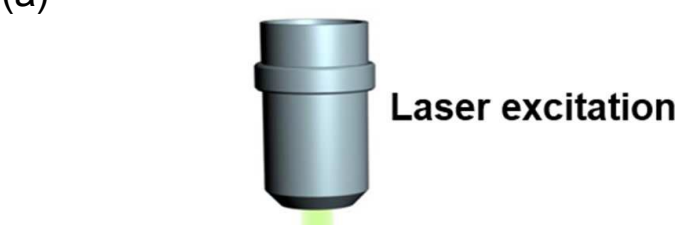

Perovskite microwire
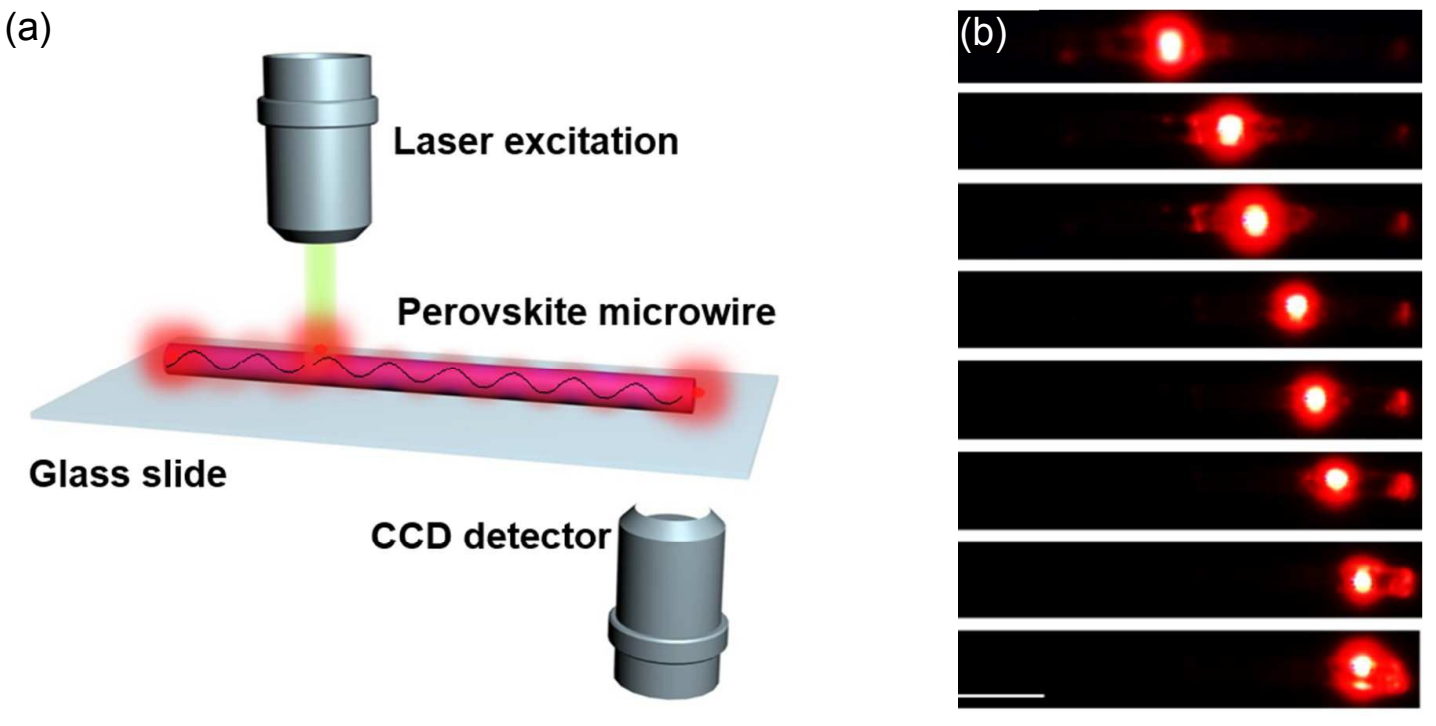

(c)

(d)
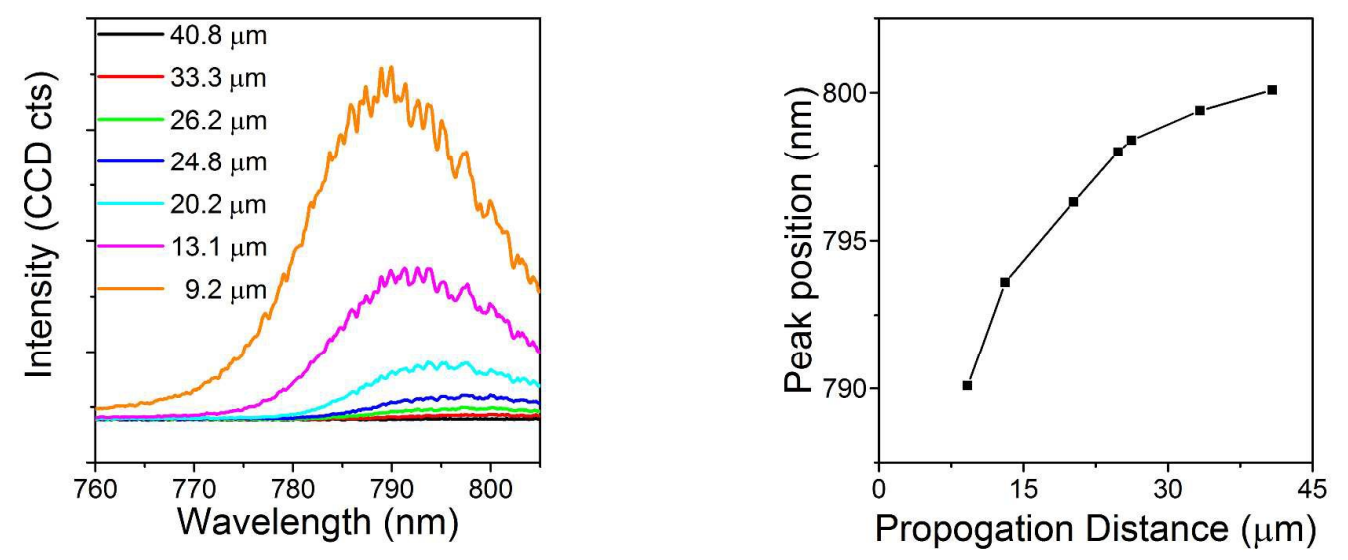

Figure 2 (a) Schematic illustration for waveguide measurement. (b) A set of dark field images of a $\mathrm{CH}_{3} \mathrm{NH}_{3} \mathrm{PbI}_{3}$ microwire. Scale bar: $10 \mu \mathrm{m}$. (c) Spatially resolved PL spectra out-coupled at right tip with different propagation distance from $9.2 \mu \mathrm{m}$ to $40.8 \mu \mathrm{m}$. (d) Out-coupled PL peak position versus propagation distance. 

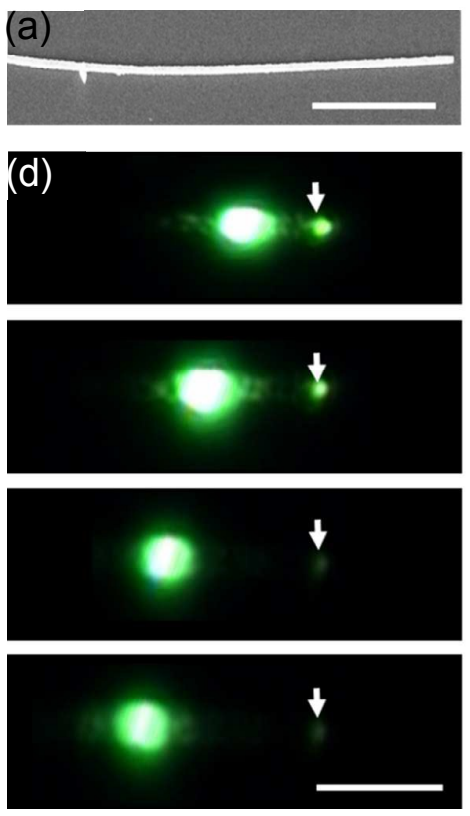

(g)

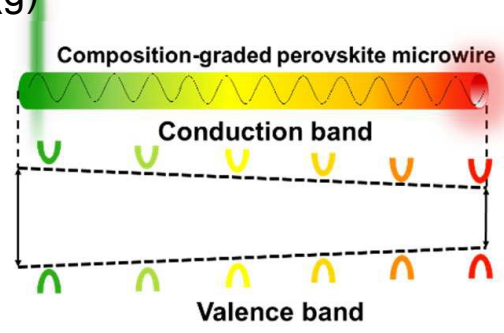

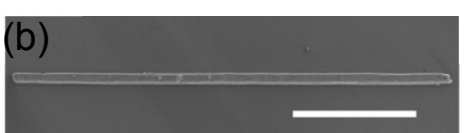
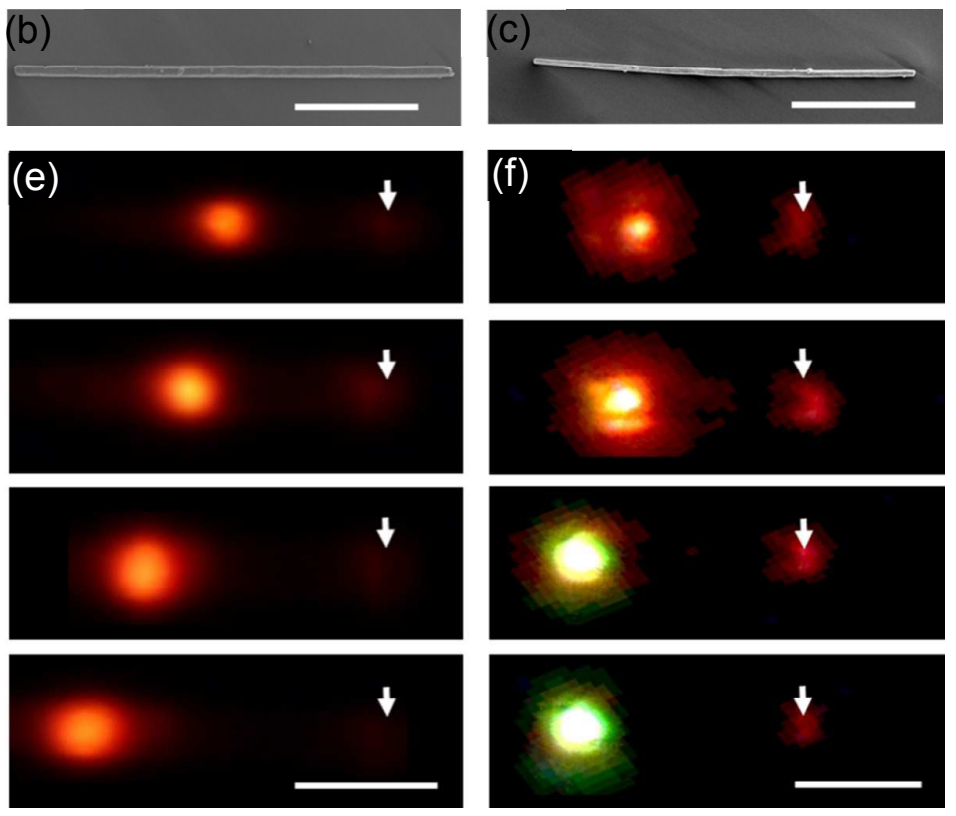

(h)

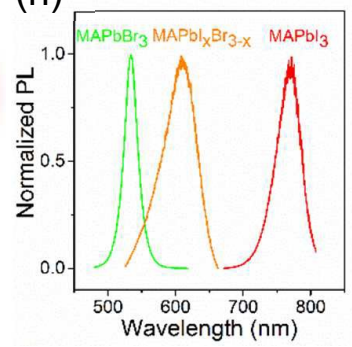

(i)

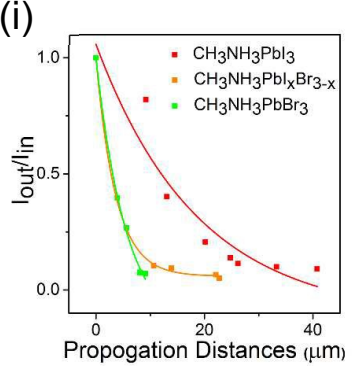

Figure 3. (a-c) SEM images of $\mathrm{MAPbBr}_{3}, \mathrm{MAPbBr}_{\mathrm{x}} \mathrm{I}_{3-\mathrm{x}}$ and composition-graded perovskite microwires. Scale bars: $20 \mu \mathrm{m}$. (d-f) Dark field PL images of $\mathrm{MAPbBr}_{3}, \mathrm{MAPbBr}_{\mathrm{x}} \mathrm{I}_{3-\mathrm{x}}$ and composition-graded perovskite microwires under local excitation. Scale bars: $5 \mu \mathrm{m}$. (g) Schematic illustrations of light propagation in a composition-graded perovskite microwire and its gradually changed bandgap along the long axis. (h) Normalized PL spectra of perovskite microwires with different compositions. (i) $I_{\text {out }} / I_{\text {in }}$ versus propagation distance and exponential fit for perovskite microwires with different compositions. 
(a)

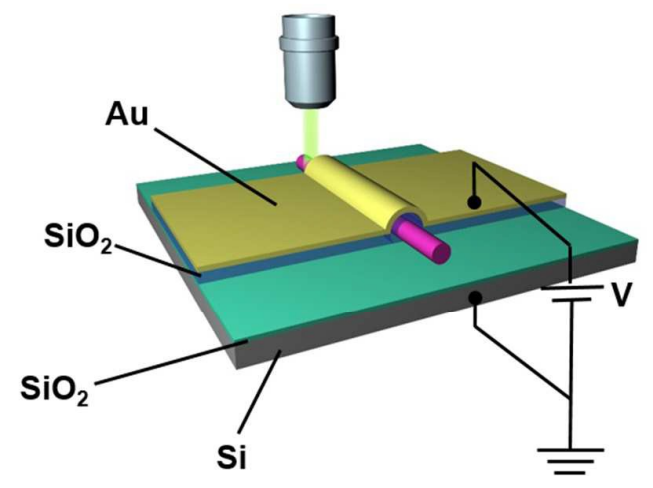

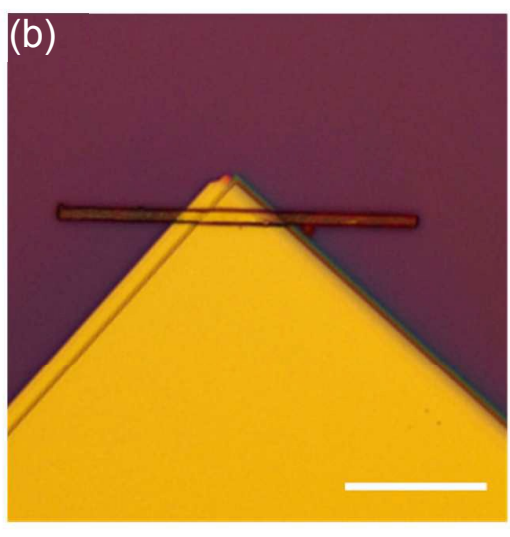

(c)

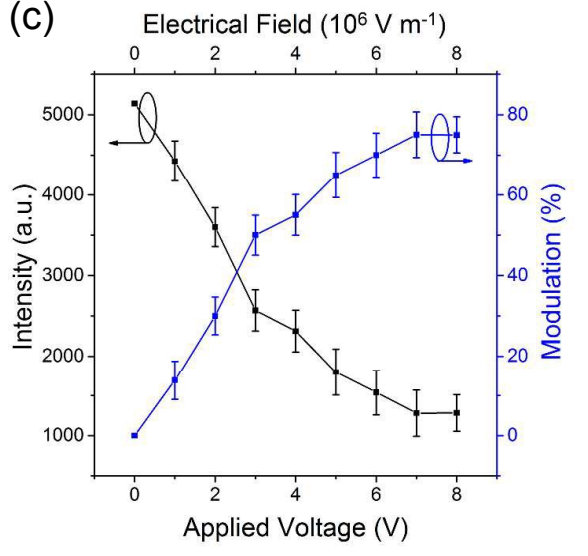

(d)

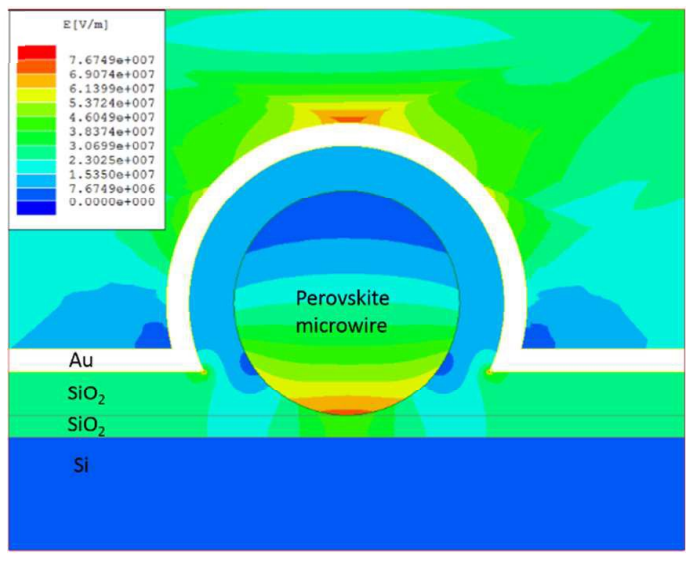

Figure 4. (a) Schematic illustration of perovskite based EOM. (b) Optical image of the EOM device. Scale bar: $10 \mu \mathrm{m}$. (c) PL intensity modulation versus applied voltage as well as local electrical field. (d) Cross-section view of electrical field distribution in the perovskite-based EOM device. The simulation was done by finite element method (applied voltage $=8 \mathrm{~V}$ ). 

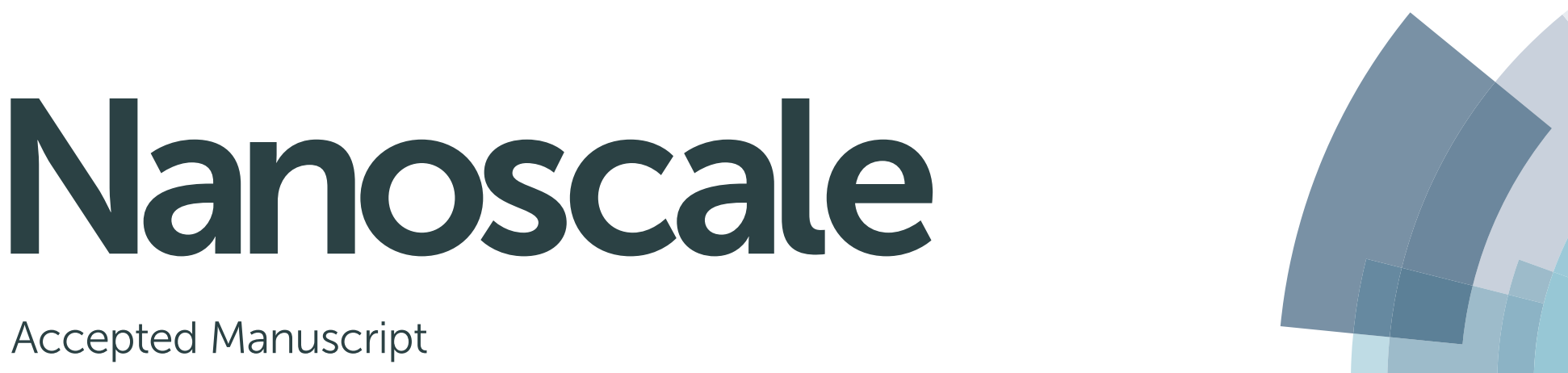

\section{Accepted Manuscript}

This article can be cited before page numbers have been issued, to do this please use: Z. Wang, J. Liu, Z. Xu, Y. Xue, L. Jiang, J. C. Song, F. Huang, Y. Wang, Y. L. Zhong, Y. Zhang, Y. Cheng and Q. Bao, Nanoscale, 2015, DOI: 10.1039/C5NR06262D.

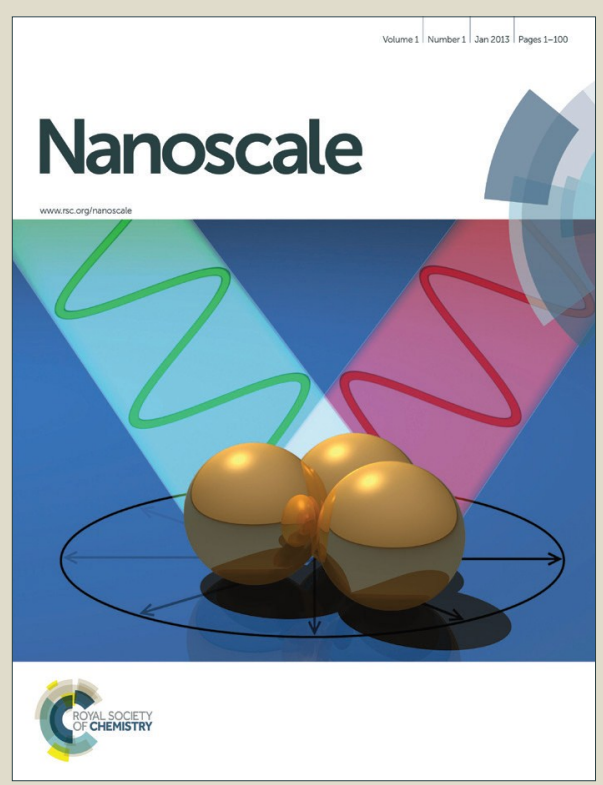

This is an Accepted Manuscript, which has been through the Royal Society of Chemistry peer review process and has been accepted for publication.

Accepted Manuscripts are published online shortly after acceptance, before technical editing, formatting and proof reading. Using this free service, authors can make their results available to the community, in citable form, before we publish the edited article. We will replace this Accepted Manuscript with the edited and formatted Advance Article as soon as it is available.

You can find more information about Accepted Manuscripts in the Information for Authors.

Please note that technical editing may introduce minor changes to the text and/or graphics, which may alter content. The journal's standard Terms \& Conditions and the Ethical guidelines still apply. In no event shall the Royal Society of Chemistry be held responsible for any errors or omissions in this Accepted Manuscript or any consequences arising from the use of any information it contains. 